MaVina Pantazara

ORCID: 0000-0002-9963-2635

Université nationale et capodistrienne d'Athènes mavinap@frl.uoa.gr

ELŻBIETA SKIBIŃSKA

ORCID: 0000-0002-3484-3984

Université de Wrocław

elzbieta.skibinska@uwr.edu.pl

\title{
LA TRADUCTION SERT-ELLE À PROPAGER LES CONNAISSANCES EN TRADUCTOLOGIE? ÉTUDES DES CAS GREC ET POLONAIS
}

\begin{abstract}
Translations have a key role in introducing new ideas, concepts and perspectives into fields which can never be seen in isolation from a wider international context, and it is often foreign theory, circulated and made available in translation, which helps to challenge established positions and open the way to key innovations ${ }^{1}$.

On croit souvent que la vie intellectuelle est spontanément internationale. Rien n'est plus faux ${ }^{2}$.
\end{abstract}

${ }^{1}$ E. Bielsa, « Some remarks on the sociology of translation: A reflection on the global production and circulation of sociological works ", European Journal of Social Theory 14(2), p. 203.

2 P. Bourdieu, "Les conditions sociales de la circulation internationale des idées », [dans :] Actes de la Recherche en Sciences Sociales, t. 145, décembre 2002, p. 3. 


\section{INTRODUCTION}

Si la traductologie étudie la traduction sous toutes ses formes et aspects, quel est le rôle de la traduction elle-même dans la diffusion des connaissances en traductologie ? La question semble d'autant plus pertinente que, même si l'anglais semble être devenu la langue des Translation Studies, des travaux traductologiques en d'autres langues continuent à être publiés. Le rayonnement de ces travaux peut être cependant limité, même si l'on admet que le «multilinguisme passif » — tel qu'il est prôné par Mary Snell-Hornby $(1999)^{3}$ — est une manière de limiter les dangers qu'entraîne la dominance de l'anglais comme langue d'échanges dans la recherche sur la traduction ${ }^{4}$. Faire entrer, par le biais de la traduction, des études publiées en grec, polonais, finnois, néerlandais, slovaque, etc. dans le circuit international de pensée traductologique semblerait ainsi une démarche naturelle, employée à côté d'autres formes de transfert des savoirs.

Cette étude s'inscrit dans deux champs de recherches plus larges - celles qui examinent le rôle de la traduction dans la dissémination des savoirs scientifiques ${ }^{5}$ et celles qui s'intéressent aux développements des connaissances en traductologie $^{6}$ - et a pour objectif d'étudier la part que joue la traduction (ou intraduction $^{7}$ ) dans la propagation des acquis en traductologie dans deux pays, la Grèce et la Pologne. Les deux peuvent être considérés comme périphériques, aussi bien par leur situation (géographique, économique, politique) en Europe, que par leur place dans la production des connaissances : il s'agit de pays (et cultures)

3 « As members of a scientific community working with different languages and cultures, translation scholars need a bilingual and bicultural proficiency similar to that expected of practising translators and interpreters [...] This would extend to passive knowledge of languages other than their mother tongue and their active working language, at least for reading purposes. "Passive multilingualism", [...] may well be the key, not only to transcultural communication among the countries of Europe, but also to a more diversified and more accurate scholarly debate in the discipline of Translation Studies worldwide » (M. Snell-Hornby, The Turns of Translation Studies, John Benjamins, Amsterdam 1999, p. 174) ; cf. aussi M. Snell-Hornby, « Is Translation Studies going Anglo-Saxon? Critical comments on the globalization of a discipline », [dans :] D. Gile, G. Hansen, N.K. Pokorn (dir.), Why Translation Studies Matters?, John Benjamins, Amsterdam 2010, p. 100.

${ }^{4}$ Ce danger peut être illustré par l'exemple de Daniel Gouadec, chercheur reconnu dans le monde francophone, qui n'a acquis une résonance internationale qu' après la traduction de son livre en anglais (D. Toudic, " Voices of Theory and Transferability: Daniel Gouadec and the 'Rennes Model' of Translation Training ", [dans :] I. Génin, I. Klitgård (dir.), Translating the Voices of Theory / La Traduction des voix de la théorie, Éditions québécoises de l'œuvre, Montréal 2015, pp. 177-200).

5 Pour ne citer que R. Schögler, Circulation of Academic Thought: Rethinking Translation in the Academic Field, Peter Lang, Berlin 2019.

${ }^{6}$ Pour ne citer que L. D’hulst, Y. Gambier (dir.), A History of Modern Translation Knowledge. Sources, Concepts, Effects, John Benjamins, Amsterdam 2018 (en particulier : A. Assis Rosa, «Forms and formats of dissemination of translation knowledge », pp. 203-214).

7 Les termes « intraduction » (importation des œuvres sous forme de traduction) et « extraduction » (exportation sous forme de traduction) sont empruntés à V. Ganne et M. Minon, « Géographies de la traduction », [dans :] F. Barret-Ducrocq (dir.), Traduire l'Europe, Payot, Paris 1992, p. 58. 
importateurs et non exportateurs. Cependant, l'organisation du monde académique propre à chacun de ces pays entraîne une position différente de la traduction, de la formation à la traduction et de la recherche traductologique ; ceci peut se manifester aussi à travers le contenu et l'utilisation des ouvrages traductologiques en tant qu'objet de l'intraduction.

Dans ce qui suit, nous commençons par donner des informations de base sur le système académique des deux pays et un bref aperçu de la situation de la formation à la traduction/traductologie et de la recherche traductologique. Dans la deuxième partie, nous présentons les résultats d'une analyse quantitative de l'intraduction, en grec et en polonais, des travaux traductologiques. Il ne suffit cependant pas qu'un livre soit publié pour qu'il soit lu et que les idées qu'il est censé véhiculer circulent effectivement ; aussi, dans la troisième partie, nous nous intéressons à l'usage didactique de ces traductions, autrement dit à la place qu'elles occupent dans les listes des lectures obligatoires ou conseillées aux étudiants qui suivent des cours en traduction ou en traductologie. Nous voulons mesurer ainsi la part que les ouvrages traduits peuvent avoir dans la diffusion des connaissances traductologiques venant des diverses traditions, pays ou centres. En effet, comme le soulignent Hanna Risku, Angela Dickinson et Richard Pircher, les compétences du traducteur, telles qu'elles sont comprises aujourd'hui, exigent, à part les connaissances linguistiques, des connaissances solides sur la traduction, ses techniques, son histoire et son rôle dans la société ${ }^{8}$. Par ailleurs, comme le constatent Ioana Balacescu et Bernd Stefanink, « $[\ldots][\mathrm{u}]$ ne didactique de la traduction est toujours fonction d'une approche théorique $»^{9}$.

\section{LA FORMATION À LA TRADUCTION ET LA RECHERCHE EN TRADUCTOLOGIE EN GRĖCE ET EN POLOGNE}

\subsection{LA FORMATION À LA TRADUCTION}

Dès 1999, plusieurs états membres de l'Union européenne, dont la Grèce et la Pologne, ont signé l'accès au Processus de Bologne, un processus de rapprochement des systèmes européens d'études supérieures. Dans les deux pays, de nouvelles législations sur les études supérieures ont suivi et ont conduit à une redéfinition et réorganisation des formations académiques. À ce jour, la Grèce n'a pas encore mis en place toutes les réformes préconisées, ce qui fait que les deux pays présentent certaines différences au niveau de l'organisation des études et des diplômes, dont rend compte, sommairement, le tableau 1.

${ }^{8}$ H. Risku, A. Dickinson, R. Pircher, « Knowledge in Translation Studies and Translation Practice: Intellectual Capital in Modern Society », [dans :] D. Gile, G. Hansen, N.K. Pokorn (dir.), Why Translation Studies Matters?, pp. 89-90.

9 I. Balacescu, B. Stefanink, « La didactique de la traduction à l'heure allemande », Meta 50(1), 2005, p. 277. 
Tableau 1. L'enseignement supérieur en Grèce et en Pologne

\begin{tabular}{|c|c|c|c|}
\hline & & \\
\hline & & Grèce & Pologne \\
\hline \multicolumn{2}{|c|}{ Nombre d'habitants (2011) } & 10816286 & 38511824 \\
\hline \multicolumn{2}{|c|}{$\begin{array}{l}\text { Nombre d'universités et } \\
\text { d'autres écoles supérieures } \\
(2019 / 2020)^{10}\end{array}$} & $\begin{array}{l}46 \text { universités et écoles } \\
\text { supérieures } \\
13 \text { départements de langues } \\
\text { et littératures étrangères } \\
1 \text { département spécialisé en }\end{array}$ & $\begin{array}{l}\text { Plus de } 400 \text { établissements } \\
\text { de l'enseignement supérieur } \\
\text { publics et privés, dont } 18 \\
\text { universités « classiques }{ }^{11}\end{array}$ \\
\hline \multirow{4}{*}{$\begin{array}{l}\text { Organisation } \\
\text { des études et } \\
\text { diplômes }\end{array}$} & Premier cycle & 4 ans, ptychio ('diplôme') & 3 ans, licencjat ('diplôme') \\
\hline & $\begin{array}{l}\text { Deuxième } \\
\text { cycle }\end{array}$ & $\begin{array}{l}1 \text { à } 2 \text { ans, metaptychiako } \\
\text { ('master') }\end{array}$ & 2 ans, magister ('master') \\
\hline & $\begin{array}{l}\text { Post-diplôme } \\
\text { (professionna- } \\
\text { lisation) }\end{array}$ & ne s'applique pas & $\begin{array}{l}\text { études podyplomowe } \\
\text { ('post-diplôme') pour les } \\
\text { titulaires de licencjat ou ma- } \\
\text { gisterium, de } 6 \text { mois à } 2 \text { ans }\end{array}$ \\
\hline & $\begin{array}{l}\text { Troisième } \\
\text { cycle }\end{array}$ & $\begin{array}{l}3 \text { à } 6 \text { ans, didaktoriko ('doc- } \\
\text { torat') pour les titulaires de } \\
\text { metaptychiako }\end{array}$ & $\begin{array}{l}4 \text { ans, doktor ('doctorat') pour } \\
\text { les titulaires de magisterium }\end{array}$ \\
\hline
\end{tabular}

Les changements survenus ont cependant eu un impact plutôt faible sur la formation universitaire à la traduction et l'interprétation. Dans chaque pays, les programmes proposés présentent des disparités aussi bien quant à leur place dans les cursus qu'à leur contenu (voir le tableau 2). Ceci reste directement lié à la situation de la profession : les compétences requises du traducteur ne sont toujours pas définies dans la législation et l'accès à la profession n'est pas régulé (à part le traducteur assermenté ${ }^{12}$ ).

\subsubsection{EN GRÈCE}

En 1979 (année de l'adhésion de la Grèce aux Communautés Européennes), le premier Centre de Traduction et d'Interprétation (Kentro Metafrasis ke Dierminias, KEMEDI) a été fondé par le Ministère de l'Éducation nationale à Corfou. En 1986, le KEMEDI a cédé sa place à un Département de langues étrangères, de traduction et d'interprétation (DFLTI) de l'Université ionienne à Corfou demeurant

${ }^{10} \mathrm{Cf}$. pour la Grèce $<$ https://www.minedu.gov.gr/ $>$ et $<$ https://eudoxus.gr $>$, et pour la Pologne $<$ https://www.gov.pl/web/nauka/uczelnie-wykazy> [consultés le 20.04.2020].

11 Par « université classique » on entend ici une université qui comprend des facultés des sciences humaines, sciences sociales, sciences exactes et sciences naturelles (et non pas des universités d'économie, d'agriculture, de médecine, etc.). Pour les besoins de ce travail, seules les universités « classiques » publiques ont été prises en compte.

12 Pour la Pologne : Ustawa z dnia 25 listopada 2004 r. o zawodzie thumacza przysiętego [Loi du 25 novembre 2004 sur la profession de traducteur assermenté]. En Grèce, les traductions assermentées sont effectuées par le service de traduction du Ministère des Affaires étrangères [Loi 4505/2017], les avocats [Loi 4194/2013] et les traducteurs diplômés du DFLTI [Décret présidentiel 169/2002]. 
jusqu'aujourd'hui le seul département universitaire à délivrer un diplôme de premier cycle (ptychio) spécialisé en traduction et en interprétation.

Pour les études de premier cycle, les deux grandes universités du pays, celle d'Athènes (dans la suite : NKUA) et celle de Thessalonique (dans la suite : AUTH), rassemblent plusieurs départements de langues et de littératures étrangères (Écoles dites " philologiques »). Alors que ceux-ci formaient traditionnellement des professeurs de langues, actuellement ils proposent de nombreux cours de traduction, théoriques et pratiques, obligatoires ou en option. Bien que leur diplôme soit unique pour tous les étudiants (sans mention d'une spécialisation par ex. en traduction ou en didactique), la plupart de ces départements prennent soin de faire figurer les services de traduction parmi leurs débouchés professionnels (par ex. dans le supplément de diplôme) ${ }^{13}$.

C'est donc seulement lors des études de deuxième cycle (metaptychiako) que les étudiants des écoles " philologiques » peuvent suivre une spécialisation en traduction. Les deux premiers programmes de master en traduction et/ou en traductologie ont fait leur apparition en 1998, à Corfou et à Athènes. Le programme « Traduction-Traductologie » (NKUA), ayant eu un parcours réussi pendant vingt ans (1998-2018), a été le fruit de la collaboration entre départements (français, anglais et allemand, NKUA) et, pendant un certain temps, entre universités (avec la participation du département d'italien et d'espagnol, AUTH) ; on peut souligner particulièrement le fait qu'il a donné lieu à un nombre important de livres traduits dans le cadre de ses cours pratiques de traduction et ensuite publiés chez différents éditeurs ${ }^{14}$. Au cours des années, les programmes de master spécialisés en traduction se sont multipliés, arrivant actuellement au nombre de neuf ${ }^{15}$, dont un (AUTH) fait partie du réseau de Master européen en traduction (EMT), en partenariat avec la DG Traduction (2014-2019 et 2019-2024).

Dans le cadre de la formation tout au long de la vie, la formation à la traduction est particulièrement florissante et ne se limite pas qu'aux universités. Une pléthore de cours, séminaires et ateliers de traduction sont offerts par des institutions diverses (instituts étrangers ${ }^{16}$, centres de traduction ${ }^{17}$ et écoles de langues privées).

13 Dans ces départements, divisés généralement en sections (reflétant l'organisation interne des études mais aussi la spécialisation de leurs enseignants-chercheurs), la traduction figure soit dans la section de Langue-Linguistique, soit dans la section de Littérature. Seuls les deux départements de langue et littérature françaises (NKUA et AUTH) ainsi que celui de langue et littérature anglaises (AUTH) ont une section de traduction à part entière ( $c f$. A. Wiedenmayer, «I didaskalia tis metafrasis sta tmimata filologias xenon glosson », [dans :] E. Lamprou, G. Floros (dir.), I didaktiki tis metafrasis ston ellinophono choro, Ellinika Grammata, Athènes 2010, pp. 177-191).

${ }^{14}$ Pour la liste complète de ses 45 publications : <http://www.translation.uoa.gr/oi-ekdoseistoy-dpms.html> [consulté le 20.04.2020].

15 Cf. D. Lamprou, I didaktiki tis diaglossikis metafrasis ston ellinophono choro, [thèse de doctorat], Université Aristote de Thessalonique, Thessalonique 2020.

16 Istituto Italiano di Cultura (à partir de 1980), Institut français d'Athènes (1981), Hellenic American Union (1990), British Council (1991), Institut Poushkin (1992), Goethe-Institut (1995), Instituto Cervantes (2002) (cf. D. Lamprou, op. cit.).

${ }^{17}$ Notons en particulier le prestigieux Centre Européen de Traduction - Littérature et Sciences de l'homme (EKEMEL), organisme privé subventionné par le Ministère de la Culture 
Les associations des traducteurs et des agences de traduction organisent elles aussi des séminaires de durée variée et sur objectifs spécifiques (traduction spécialisée, technique, littéraire, audiovisuelle, traduction assistée par ordinateur, etc.).

\subsubsection{EN POLOGNE}

Les futurs traducteurs ont pu se former, dès 1963, à Wyższe Studium Języków Obcych (École Supérieure des Langues Étrangères) auprès de l'Université de Varsovie. La formation, novatrice pour son époque, durait quatre ans ; elle était centrée sur les compétences pratiques de traduction non littéraire, de deux langues étrangères, et préparait au travail de traducteur et interprète. Elle conduisait à un diplôme d'études supérieures, mais non pas à celui de magister (celui-ci était délivré au bout de cinq ans d'études, finissant par un mémoire). Elle a été fermée au début des années 1970 et son activité a été reprise, à partir de 1973, par Instytut Lingwistyki Stosowanej (Institut de Linguistique appliquée, ILS). Pendant près de vingt ans, il a été le seul centre universitaire à former des traducteurs ${ }^{18}$.

Dans les années 1990, à la suite des changements politiques de 1989, des réformes de l'enseignement supérieur ont ouvert de nouvelles possibilités de formation à la traduction dans les facultés de langues et civilisations étrangères (filologia) qui, jusqu'ici, comme en Grèce, formaient principalement des enseignants de langues. En réponse à une forte demande du marché, elles ont commencé à proposer à leurs étudiants des cours de traduction. Des formations post-diplôme pour les titulaires du diplôme de magister ont été créées également, telles les Podyplomowe Studia dla Tłumaczy (Études post-diplôme pour Traducteurs) de l'Université Jagellonne de Cracovie (1995), ou les Interdyscyplinarne Podyplomowe Studia Kształcenia Tłumaczy Instytutu Lingwistyki Stosowanej (Études interdisciplinaires de Formation de Traducteurs de l'ILS) (1998) et de nombreuses autres formations, parfois éphémères ${ }^{19}$.

Divers enseignemements sont offerts actuellement aux futurs traducteurs:

(1) Programmes de deuxième cycle en langue et civilisations étrangères (filologia) qui, dans le cadre des cursus traditionnellement appelés « philologiques », proposent une spécialisation «traduction ». Pour obtenir le diplôme de magister, les étudiants doivent présenter un mémoire qui montre les résultats de leur recherche menée dans le cadre d'un séminaire, sous la direction

grec (2001-2012), qui a formé toute une génération de traducteurs et qui a, entre autres, publié une revue en ligne, Apiliotis, et même créé des prix de traduction ( $c f$. M. Volkovitch, «Quand les Grecs donnent l'exemple », Translittérature 51, 2017, pp. 16-17).

18 A. Kopczyński, «Wyższe Studium Języków Obcych Uniwersytetu Warszawskiego — pierwsza szkoła thumaczy w Polsce (1963-1972) », [dans :] K. Hejwowski, A. Szczęsny, U. Topczewska (dir.), 50 lat polskiej translatoryki, ILS, Varsovie 2009, pp. 53-55.

19 M. Tryuk, « Początki przekładu ustnego w Polsce. Pierwszy etap profesjonalizacji rynku », [dans :] K. Hejwowski, A. Szczęsny, U. Topczewska (dir.), 50 lat polskiej translatoryki, p. 62. 
d'un professeur ; souvent, mais pas systématiquement, les étudiants qui suivent la spécialisation «traduction » présentent un mémoire sur un sujet traductologique.

(2) Programmes de traduction littéraire dans le cadre des études de deuxième cycle en polonais, spécialité traduction (UJ, UAM).

(3) Programmes de master en traduction (ILS) ; certains appartiennent au réseau EMT.

(4) Études post-diplôme pour les titulaires de diplôme de licencjat ou magister; celles-ci, suite au rétrécissement du marché du travail, se font de moins en moins nombreuses.

(5) Il existe aussi des cours d'initiation à la traduction et/ou la traductologie dans le cadre du premier cycle en langue et civilisations étrangères.

Quelle que soit la forme, la durée et le contenu de la formation, son programme contient une composante de caractère théorique qui exige des étudiants une connaissance de travaux traductologiques.

En dehors du milieu académique, les associations des traducteurs et des agences de traduction offrent des formations de toute sorte et de durée variable, dans lesquelles l'accent est mis principalement sur la pratique de la traduction.

Tableau 2. Formation académique à la traduction en Grèce et en Pologne

\begin{tabular}{|c|c|c|}
\hline & Grèce & Pologne \\
\hline $\begin{array}{l}1^{\text {er }} \text { cycle } \\
\text { Études avec } \\
\text { diplôme spécial }\end{array}$ & DFLTI & ILS \\
\hline $\begin{array}{l}1^{\text {er }} \text { cycle } \\
\text { Études sans } \\
\text { diplôme spécial }\end{array}$ & $\begin{array}{l}\text { modules de traduction dans } \\
\text { les départements des langues } \\
\text { étrangères (interlinguistique) } \\
\text { et du grec (intralinguistique) } \\
\text { — sans mention de spécialisation }\end{array}$ & $\begin{array}{l}\text { modules de spécialisation en traduc- } \\
\text { tion dans les départements de langues } \\
\text { étrangères (filologia) et du polonais } \\
\text { (polonistyka) } \\
\text { — avec mention de la spécialisation } \\
\text { dans le supplément de diplôme }\end{array}$ \\
\hline $\begin{array}{l}2^{\mathrm{e}} \text { cycle } \\
\text { Études avec } \\
\text { diplôme spé- } \\
\text { cial }^{20}\end{array}$ & $\begin{array}{l}9 \text { (NKUA, AUTH, DFLTI) } \\
\text { dont } 1 \text { EMT (AUTH) }\end{array}$ & $\begin{array}{l}\text { ILS — master EMT, EMCI } \\
\text { UAM - master EMT } \\
\text { UJ, chaire de la traductologie - } \\
\text { EMT } \\
\text { UP Cracovie, philologie anglaise - } \\
\text { EMT }\end{array}$ \\
\hline $\begin{array}{l}2^{\mathrm{e}} \text { cycle } \\
\text { Études avec ou } \\
\underline{\text { sans diplôme }} \\
\text { spécial }\end{array}$ & $\begin{array}{l}\text { séminaires aidant à rédiger un mé- } \\
\text { moire en Traduction-Traductologie } \\
\text { pour les programmes avec diplôme } \\
\text { spécial seulement }\end{array}$ & $\begin{array}{l}\text { séminaires aidant à rédiger un mé- } \\
\text { moire en Traduction-Traductologie } \\
\text { pour les programmes avec ou sans } \\
\text { diplôme spécial }\end{array}$ \\
\hline $\begin{array}{l}\text { Études } \\
\text { post-diplôme }\end{array}$ & ne s'applique pas & $\begin{array}{l}\text { oui } \\
\text { studia podyplomowe }\end{array}$ \\
\hline
\end{tabular}

${ }^{20}$ Avec mention du label EMT pour la période 2019-2024. 


\subsection{LA RECHERCHE EN TRADUCTOLOGIE}

Nous mettons, par la suite, en évidence la place accordée dans les deux pays à la recherche en traductologie, science qui étudie le phénomène de la traduction sous ses multiples formes et selon des approches variées (linguistique, culturelle, sociologique, historique...).

\subsubsection{EN GRÈCE}

La traduction, tant intralinguistique (du grec ancien) qu'interlinguistique a, depuis bien longtemps, fait l'objet des réflexions contenues dans les paratextes des traductions et les travaux des philologues ${ }^{21}$. Au cours des dernières décennies, le développement de la recherche traductologique se systématise, ce qui se reflète dans le nombre croissant des enseignants-chercheurs ayant dans le descriptif de leur poste la théorie de la traduction (metafraseologia) qui, en 2018, étaient au nombre de 13 (face aux 20 qui enseignent la pratique ou la traduction en général) $)^{22}$. Les principaux éléments qui ont contribué à la systématisation des recherches traductologiques sont les suivants :

(1) Les revues : les premiers travaux traductologiques sont parus dans des revues philologiques et littéraires ou dans des rubriques ou des numéros spéciaux sur la traduction, mais surtout dans la revue thématique, annuelle, non universitaire, Metafrasi (1995-2007), qui a publié de manière plus systématique des articles de chercheurs grecs, mais aussi des textes de traductologues étrangers, surtout français, tels Jean-René Ladmiral ou Georges Mounin, traduits pour la première fois en grec.

(2) Les colloques et la publication des actes : les premiers colloques dans les années 1990 avaient comme axe principal la traduction dans l'UE. Au fil des années, le nombre de communications sur la traduction, notamment dans des colloques de linguistique appliquée, augmente. Depuis 2006, les traductologues hellénophones (grecs et chypriotes) ont leur colloque biannuel à Thessalonique, organisé par la Section de Traduction du Département de langue et littérature françaises (AUTH).

(3) La Société hellénique de Traductologie (EEM), fondée en 2011 pour rassembler les chercheurs grecs et promouvoir la recherche en traductologie

${ }^{21}$ Pour la traduction intralinguistique, $c f$. entre autres : I.Th. Kakridis, To metafrastiko provli$m a$, Vivliopolion tis Estias, Athènes 1984 [première édition : 1936]; D. Maronitis, «Intralingual Translation. Genuine and False Dilemmas ", [dans :] A. Lianeri, V. Zajko (dir.), Translation and the Classic: Identity as Change in the History of Culture, Oxford University Press, Oxford 2008, pp. 367-386. Pour un aperçu global de l'histoire de la traduction en Grèce, $c f$. S. Grammenidis, G. Floros, «The Greek-speaking Tradition », [dans :] Y. Gambier, U. Stecconi (dir.), A World Atlas of Translation, John Benjamins, Amsterdam 2019, pp. 323-340.

22 Conformément à la liste des enseignants-chercheurs des universités grecques, diffusée par le Ministère de l'Éducation nationale (août 2018). 
dans plusieurs langues pratiquées. À ce jour, elle compte 60 membres et plus de 1300 abonnés sur sa page facebook.

(4) La mise en ligne des données : une base de données bibliographiques en traductologie, réalisée sous la direction de Simos Grammenidis, rassemble tous les travaux publiés par des traductologues hellénophones jusqu'en 2013 (715, dont 45 thèses $)^{23}$.

(5) La présence internationale des chercheurs en traductologie grecs de plus en plus forte et visible à l'étranger : on peut mentionner, à titre indicatif, la participation des universités grecques à des projets de recherche européens (par ex. TraMOOC - Translation for Massive Open Online Courses ou TRAMIG Training newly arrived migrants for community interpreting and intercultural mediation ${ }^{24}$ ), la présence de chercheurs grecs dans des conseils d'administration des associations internationales (par ex. EST - European Society for Translation Studies), la participation des professeurs grecs à des jurys d'évaluation de thèses à l'étranger (par ex. en Roumanie ou en Espagne) et les contributions grecques dans des publications spécialisées internationales ${ }^{25}$.

\subsubsection{EN POLOGNE}

Les années 1990, qui ont vu la forte augmentation des formations à la traduction, ont été marquées aussi par une croissance spectaculaire des travaux de recherche sur la traduction. Objet de recherche déjà auparavant ${ }^{26}$, elle a commencé à attirer l'attention de nombreux chercheurs, de diverses générations. Les résultats de leurs analyses, publiés dans des livres ou de nouvelles revues ou collections spécialisées, ou encore présentés lors de nombreux colloques (certains, devenus cycliques, sont un élément important de la traductologie polonaise), témoignent de l'existence, en Pologne, de la traductologie. Ou plutôt des chercheurs-traductologues; dans des textes de loi qui régulent et organisent la recherche, la traductologie comme discipline est absente ${ }^{27}$.

${ }^{23}$ Disponible sur : <http://niobe.frl.auth.gr/hstbibliography/> [consulté le 20.04.2020].

${ }^{24} \mathrm{La}$ description du projet TraMOOC : <http://di.ionio.gr/en/european-projects/tramooc-2/> et du projet TRAMIG : <https://tramig.eu/> [consultés le 20.04.2020].

25 Pour ne citer que les plus récentes : T. Dimitroulia, D. Goutsos (dir.), inTRAlinea, Special Issue: Corpora and Literary Translation, 2017 ; S. Grammenidis, G. Floros, op. cit. ; M. Pantazara, E. Tziafa (dir.), Atelier de Traduction 31-32 (Dossier thématique : Dénomination et traduction), 2019 ; P. Nicolaou (dir.), Encounters in Greek and Irish Literature, Cambridge Scholars Publishing, 2020.

${ }^{26}$ Cf. P. de Bończa Bukowski, M. Heydel, Polish Translation Studies in Action. Concepts Methodologies - Applications. A Reader, Peter Lang, Berlin 2019.

27 Cf. E. Skibińska, « Between Richness and 'Non-existence': Polish Translation Researchers as a Community », [dans :] K. Taivalkoski-Shilov, L. Tiittula, M. Koponen (dir.), Communities in Translation and Interpreting, Éditions québécoises de l'œuvre, Montréal 2017, pp. 253-274. 
La conséquence directe en est l'impossibilité de délivrer des grades ou titres (de doctorat, d'habilitation à diriger la recherche) dans cette discipline. Cette situation contribue aussi à la pérennisation du " profilement » angliciste, germaniste, slaviste ou romaniste de la recherche, lié à la meilleure connaissance des acquis traductologiques relevant des traditions anglosaxonne, allemande, française etc., publiés le plus souvent dans les langues correspondantes ${ }^{28}$, auquel s'ajoute une sorte de séparatisme linguistique : on ne cherche pas à connaître les travaux écrits dans une langue autre que sa langue de travail ou l'anglais.

Tableau 3. Recherche en traductologie en Grèce et en Pologne

\begin{tabular}{|c|c|c|c|}
\hline & \multirow{3}{*}{$\begin{array}{l}\text { Grèce } \\
2 \text { revues : } \mathrm{mTm}^{29}, \mathrm{Me} \text { - } \\
\text { tafrasi }(1995-2008) \\
\text { numéros thématiques } \\
\text { dans d'autres revues }\end{array}$} & \\
\hline & & & \multirow[t]{2}{*}{ Pologne } \\
\hline \multirow{3}{*}{$\begin{array}{l}\text { Formes d'insti- } \\
\text { tutionnalisation }\end{array}$} & $\begin{array}{l}\text { Revues } \\
\text { et collections }\end{array}$ & & \\
\hline & Colloques cycliques & oui ( 1 seul $)$ & oui (plus de 6) \\
\hline & Associations & $\begin{array}{l}\text { EEM - Elliniki Eteria } \\
\text { Metafraseologias [Hel- } \\
\text { lenic Society for Trans- } \\
\text { lation Studies] } \\
\text { (depuis 2011) }\end{array}$ & $\begin{array}{l}\text { CTER - Konsorcjum } \\
\text { do Badań nad Edukacją } \\
\text { Tłumaczy [Consortium } \\
\text { for Translation Educa- } \\
\text { tion Research] } \\
\text { (depuis 2013) }\end{array}$ \\
\hline \multirow{4}{*}{$\begin{array}{l}\text { Reconnaissance } \\
\text { officielle de la } \\
\text { discipline }\end{array}$} & $\begin{array}{l}\text { inscrite sur la liste } \\
\text { ministérielle des } \\
\text { disciplines }\end{array}$ & non & non \\
\hline & $\begin{array}{l}\text { indiquée dans la } \\
\text { formulation des } \\
\text { titres des concours } \\
\text { pour des postes } \\
\text { universitaires }\end{array}$ & oui & non \\
\hline & $\begin{array}{l}\text { mentionnée dans } \\
\text { les appellations des } \\
\text { diplômes }\end{array}$ & $\begin{array}{l}\text { oui } \\
\text { (seulement pour } \\
\text { les } 9 \text { metaptychiako) }\end{array}$ & $\begin{array}{l}\text { oui } \\
\text { (seulement pour } \\
\text { les 4 EMT) }\end{array}$ \\
\hline & $\begin{array}{l}\text { traduction comme } \\
\text { activité reconnue } \\
\text { lors des évaluations } \\
\text { des chercheurs et } \\
\text { des universitaires }\end{array}$ & oui & non \\
\hline
\end{tabular}

28 J. Żmudzki, « Problemy, zadania i wyzwania translatoryki », Lingwistyka Stosowana 1, 2009, pp. 41-60.

${ }^{29}$ La revue $m T m$ (i.e. minor translating major, major translating minor, minor translating minor), malgré son nom, ne publie que des articles rédigés en anglais, français, allemand, italien et espagnol (et non pas en grec). 


\subsection{BILAN}

Les tableaux 2 et 3 ci-dessus réunissent des données qui permettent un bilan comparatif de la situation de la formation à la traduction et de la recherche traductologique dans les deux pays.

Ainsi, l'intérêt accru pour la traduction, en tant qu'activité professionnelle, est reflété dans le développement des études spécialisées, ce qui s'inscrit dans le contexte général, européen et mondial, dû à l'influence du marché et à la constante augmentation de la demande de traductions. Cependant, si en Pologne se dessine un « centre » de la formation et de la recherche qu'est le milieu académique avec ses diverses activités - un nombre relativement plus important de revues spécialisées (attachées à des universités), de colloques cycliques et de programmes d'études EMT - la Grèce connaît plus d'initiatives venues de la " périphérie », avec une plus grande dispersion des pôles de la formation et du développement d'une pensée traductologique (comme le rôle de la revue-phare Metafrasi ou du Centre EKEMEL). Malgré cela, il s'avère que, dans les deux pays, le statut de la traductologie en tant que discipline distincte et autonome reste encore incertain.

\section{PRÉSENCE ÉDITORIALE DES OUVRAGES TRADUCTOLOGIQUES TRADUITS EN GRÈCE ET EN POLOGNE}

\subsection{CONSTITUTION DES LISTES DES OUVRAGES RECENSÉS}

La traduction est comprise ici comme publication sous forme de livre, résultat de l'activité éditoriale qui conditionne le fonctionnement public — la diffusion - du texte. Nous nous intéressons au livre non seulement parce que dans la production scientifique en sciences humaines et sociales il occupe toujours une place centrale, mais aussi parce que, comme objet de l'activité éditoriale, il constitue une catégorie relativement facile à identifier et décrire ${ }^{30}$.

Les sources des données étant de nature différente pour chaque pays (voir le tableau 4), nous avons défini des critères communs et les avons affinés dans les analyses du corpus respectivement grec et polonais.

Le premier critère commun appliqué ici est celui de la langue de publication : grec ou polonais ; le suivant est celui de l'appartenance de l'ouvrage à la discipline de traductologie. En nous appuyant sur la définition proposée par Mathieu Guidère, nous considérons que « l'objet de la traductologie est bien la traduction dans toutes ses manifestations. Qu'il s'agisse de traduction orale ou écrite, géné-

30 Cf. J. Heilbron, « Book translations as a cultural world-system », European Journal of Social Theory 2(4), 1999, pp. 429-444; A. Pym, S. Poupaud, E. Torres-Simón, « Finding Translations. On the Use of Bibliographical Databases in Translation History », Meta 54(2), 2009, pp. 264-278. 
rale ou spécialisée, le traductologue réfléchit sur toutes les formes d'intervention du traducteur $»^{31}$. Sont donc traités comme traductologiques les ouvrages qui ont pour objet « la traduction dans toutes ses manifestations », ce qui permet de prendre en considération aussi bien les travaux hautement théoriques sur la nature de la traduction, que les guides pratiques de traduction entre deux langues données.

Tableau 4. Constitution des listes des ouvrages traduits — sources de données

\begin{tabular}{|c|c|}
\hline Grèce & Pologne \\
\hline $\begin{array}{l}\text { catalogues en ligne de : } \\
\text { (1) Bibliothèque nationale grecque } \\
\text { (2) Centre national du livre, base BiblioNet } \\
\text { (3) Base de données bibliographiques } \\
\text { en traductologie }\end{array}$ & $\begin{array}{l}\text { catalogues en ligne de : } \\
\text { (1) Bibliothèque de l'Université de Wrocław } \\
\text { (2) Bibliothèque Jagellonne } \\
\text { (3) Bibliothèque nationale polonaise }\end{array}$ \\
\hline \multicolumn{2}{|c|}{$\begin{array}{l}\text { données recoupées par la consultation du WorldCat, } \\
\text { des sites Internet des éditeurs et/ou des livres }\end{array}$} \\
\hline $\begin{array}{l}\text { liste grecque : } 24 \text { ouvrages traduits } \\
\text { (dont une anthologie) }\end{array}$ & $\begin{array}{l}\text { liste polonaise : } 18 \text { ouvrages traduits } \\
\text { (dont une anthologie) }\end{array}$ \\
\hline
\end{tabular}

Puisque, par la suite, nous nous occupons des traductions de tels ouvrages et que la traduction est comprise ici comme un livre publié en tant que traduction, un deuxième critère a été appliqué : la présence, dans la description bibliographique, de la mention przekład/ttumaczenie $z$ ou metafrasi ('traduction de') et, généralement, du nom du traducteur.

Après la constitution des listes des traductions dans chacune des deux langues, une conjecture de départ s'est vue confirmée : leurs constituants présentent un caractère hétérogène. Pour pouvoir les organiser de manière systématique, nous avons procédé à l'application des trois critères suivants :

(a) la forme de publication : livre d'un auteur, ouvrage collectif, anthologie ;

(b) le contenu de la publication : manuel (synthèse), ouvrage de recherche (monographique), guide, encyclopédie ou dictionnaire, travail relevant de la réflexion préscientifique sur la traduction ${ }^{32}$;

(c) le public visé : spécialistes (chercheurs, étudiants, professionnels), non spécialistes (vulgarisation).

Ceci a permis de classer les ouvrages analysés dans cinq catégories, toutes présentes dans les deux listes, quoiqu'en quantités différentes (graphiques 2a et 2b) :

${ }^{31}$ M. Guidère, Introduction à la traductologie, De Boeck, coll. « Traducto », Bruxelles 2010, p. 12.

32 L'expression « réflexion préscientifique » renvoie aux ouvrages portant sur la traduction (considérée aussi bien empiriquement, comme une pratique individuelle, que, avec un essai de théorisation, comme un phénomène ayant un rôle important dans l'histoire de l'humanité) parus avant la première moitié du $\mathrm{XX}^{\mathrm{e}}$ siècle et la naissance de la discipline scientifique appelée traductologie. 
- ouvrages de recherche,

- ouvrages didactiques,

- ouvrages préscientifiques,

— ouvrages de vulgarisation scientifique,

- anthologies.

\subsection{RÉSULTATS DE L'EXAMEN DES LISTES \\ DES OUVRAGES TRADUITS}

Comme le montre le tableau 4 ci-dessus, avec 23 ouvrages traduits en grec, 17 en polonais, et une anthologie dans chacune des deux langues, les résultats de nos recherches s'avèrent plutôt maigres. Ils permettent cependant de faire des observations comparatives de la situation en Grèce et en Pologne et de répondre aux questions portant sur les auteurs et les titres traduits, la langue de l'original, les éditeurs et les traducteurs des ouvrages intraduits, et la chronologie et la fréquence de leur publication.

\subsubsection{CHRONOLOGIE DE L'INTRADUCTION}

L'examen des dates de parution des ouvrages traduits permet de constater que même si deux ouvrages sont parus en polonais dans les années 1970 et deux en grec dans les années 1990, c’est seulement dans les années 2000 que les éditeurs commencent à manifester un intérêt plus affirmé pour cette discipline.

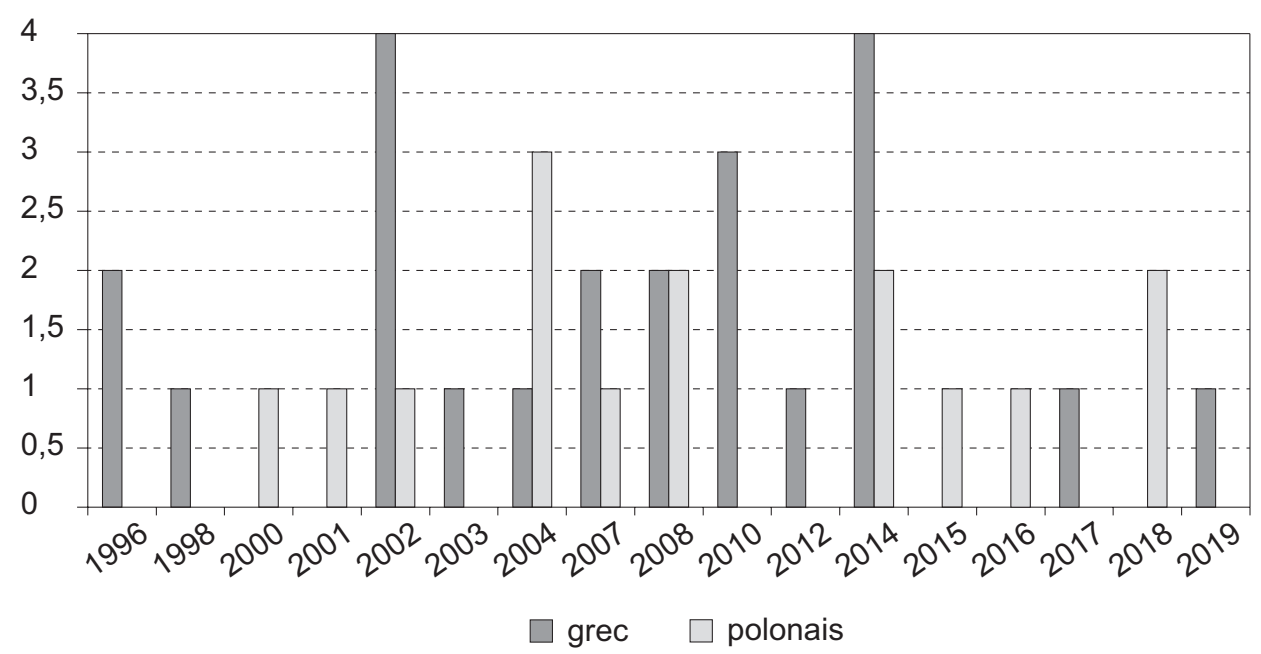

Graphique 1. Nombre de traductions, selon l'année (à partir de 1990) 


\subsubsection{CONTENU DE L'INTRADUCTION}

Les graphiques $2 \mathrm{a}$ et $2 \mathrm{~b}$, qui rendent compte du contenu de l'intraduction, montrent que le nombre d'ouvrages traduits en grec est plus élevé dans toutes les catégories à part celle d' « ouvrages préscientifiques ». En effet, on peut être frappé de la présence, en polonais, des essais d'Alexander Fraser Tytler, Matthew Arnold, Francis Newman, publiés par la Maison d'édition de l'Université de Gdańsk, dans la collection « Europejscy klasycy sztuki przekładu od XIV do XIX wieku » ['Classiques européens de l'art de traduire du XIV $\mathrm{X}^{\mathrm{e}}$ au XIX ${ }^{\mathrm{e}}$ siècle'] ; il s'agit cependant d'une initiative du traducteur de ces ouvrages. Nous y classons aussi les notes de Karl Dedecius, publiées en polonais en 1974 par Wydawnictwo Literackie, et surtout Die Aufgabe des Übersetzers de Walter Benjamin, paru en grec sous forme d'un ouvrage à part ${ }^{33}$.

En ce qui concerne les ouvrages didactiques, on constate d'abord l'existence d'un seul élément commun : le dictionnaire de Terminologie de la traduction, « vocabulaire fondamental de l'enseignement pratique de la traduction », pour citer les auteurs de la Présentation de l'ouvrage ${ }^{34}$. À part cela, on observe une différence de poids : autant les ouvrages traduits en grec sont des manuels ou introductions à la traductologie (tels de Jeremy Munday et de Jenny Williams \& Andrew Chesterman), autant les quatre traductions en polonais sont des guides pratiques d'interprétation (d'Andrew Gillies et de Jean-François Rozan), publiés sous forme de petits volumes bilingues, anglais et polonais, par la Société Cracovienne de Promotion de la Linguistique Tertium, dans la collection « Język a komunikacja » ['Langue et communication'].

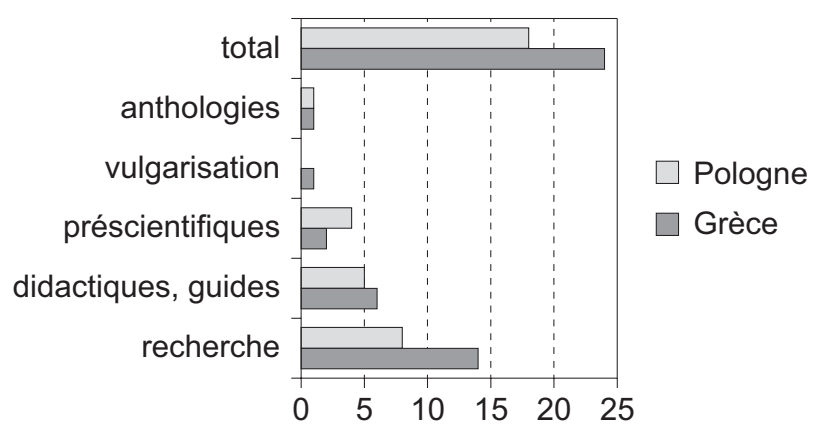

Graphique 2a. Nombre d'ouvrages traduits, selon la catégorie

${ }^{33}$ En polonais, ce texte fait partie d'un volume des travaux du philosophe (dans deux traductions différentes). Les principes appliqués dans la constitution des données n'ont pas permis d'inclure ces deux traductions dans notre liste d'ouvrages traductologiques traduits sous forme de livre.

34 J. Delisle, H. Lee-Jahnke, M.C. Cormier (dir.), Terminologie de la traduction, John Benjamins, Amsterdam 1999, p. 2. 


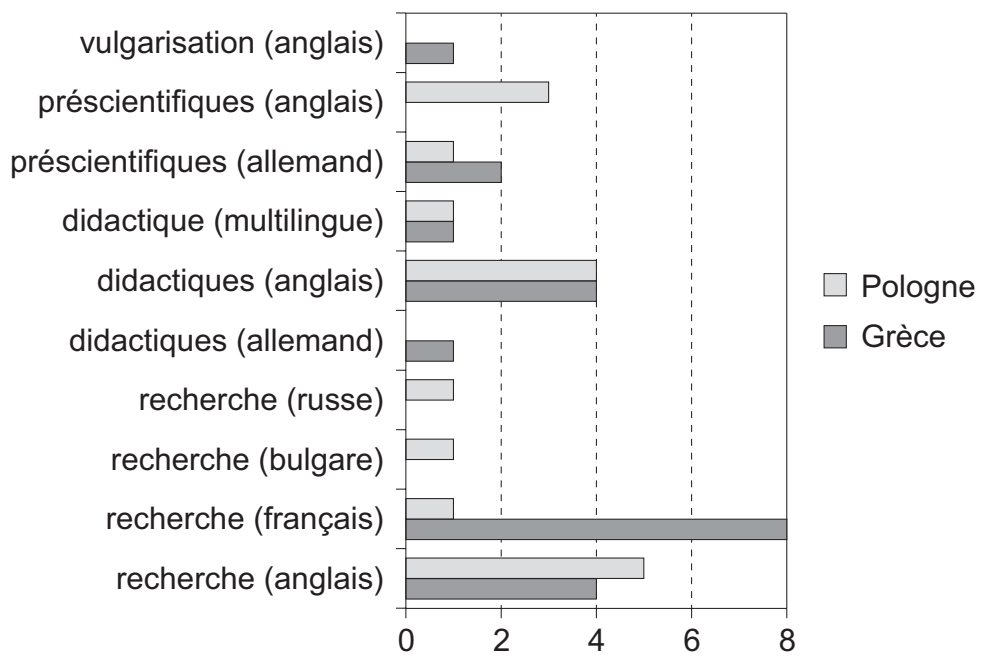

Graphique 2b. Nombre d'ouvrages traduits, selon la catégorie et la langue

La catégorie " ouvrages de recherche » présente elle aussi des différences considérables (graphiques $2 \mathrm{a}, 2 \mathrm{~b}$, tableau 5). La première concerne le nombre de langues sources : deux pour le grec, quatre pour le polonais. Parmi les traductions polonaises, on remarque un ouvrage traduit du bulgare - la seule langue périphérique dans nos inventaires ; il s'agit cependant de l'ouvrage sur la traduction humaine et la traduction mécanique, publié par Alexandre Ljudskanov dans les années 1960, traduit en polonais en 1973 et jamais réédité. Une autre différence frappante est celle de la part du français comme langue source : huit ouvrages traduits en grec face à un seul en polonais. Ainsi, les chercheurs et étudiants grecs ne parlant pas le français ont la possibilité de connaître les idées de Georges Mounin, Antoine Berman, Jean-René Ladmiral — personnages phares de la traductologie française - qui, pour leurs homologues polonais, restent toujours inaccessibles.

Tableau 5. Ouvrages traduits, catégorie « ouvrages de recherche»

\begin{tabular}{|l|l|l|c|}
\hline \multicolumn{3}{|c|}{ I. OUVRAGES TRADUITS DE L'ANGLAIS } \\
\hline \multirow{2}{*}{ Auteur } & \multicolumn{3}{c|}{ Ouvrages traduits en grec } \\
\cline { 3 - 4 } & \multicolumn{3}{|c|}{ Année de la parution } \\
\cline { 3 - 4 } \multicolumn{3}{|c|}{ original } & traduction \\
\hline Umberto Eco & Experiences in Translation & 2001 & 2003 \\
\hline Michael Cronin & Translation and Globalization & 2003 & 2007 \\
\hline Edwin Gentzler & Contemporary Translation Theories & 1993 & 2012 \\
\hline Christiane Nord & Translating as a Purposeful Activity & 1997 & 2014 \\
\hline
\end{tabular}


Tableau 5. Ouvrages traduits... (suite)

\begin{tabular}{|c|c|c|c|}
\hline \multicolumn{4}{|c|}{ I. OUVRAGES TRADUITS DE L'ANGLAIS } \\
\hline \multirow{2}{*}{ Auteur } & \multirow{2}{*}{ Titre(s) } & \multicolumn{2}{|c|}{ Année de la parution } \\
\hline & & original & traduction \\
\hline \multicolumn{4}{|c|}{ Ouvrages traduits en polonais } \\
\hline Elżbieta Tabakowska & $\begin{array}{l}\text { Cognitive Linguistics and Poetics } \\
\text { of Translation }\end{array}$ & 1993 & 2001 \\
\hline Ernst-August Gutt & Modes of Translation and Cultural Distance & 1989 & 2004 \\
\hline Theo Hermans & The Conference of the Tongues & 2007 & 2015 \\
\hline \multicolumn{4}{|c|}{ Ouvrages traduits en grec et en polonais } \\
\hline George Steiner & After Babel & 1975 & $\begin{array}{l}2000(\mathrm{pl}) \\
2004(\mathrm{gr})\end{array}$ \\
\hline Michael Cronin & Translation in the Digital Age & 2013 & $\begin{array}{l}2016(\mathrm{pl}) \\
2019(\mathrm{gr})\end{array}$ \\
\hline \multicolumn{4}{|c|}{ II. OUVRAGES TRADUITS DU FRANÇAIS } \\
\hline \multirow{2}{*}{ Auteur } & \multirow{2}{*}{ Titre(s) } & \multicolumn{2}{|c|}{ Année de la parution } \\
\hline & & original & traduction \\
\hline \multicolumn{4}{|c|}{ Ouvrages traduits en grec } \\
\hline Jacques Derrida & $\begin{array}{l}\text { Témoignage et traduction - Survivre en } \\
\text { poète [conférence en Grèce] }\end{array}$ & 1995 & 1996 \\
\hline Georges Mounin & Les belles infidèles & 1955 & 2002 \\
\hline Georges Mounin & Les problèmes théoriques de la traduction & 1963 & 2002 \\
\hline Antoine Berman & L'auberge du lointain & 1991 & 2002 \\
\hline Jean-René Ladmiral & Traduire : théorèmes pour la traduction & 1979 & 2007 \\
\hline Daniel Gouadec & Profession : Traducteur & 2002 & 2008 \\
\hline Daniel Gouadec & Faire traduire & 2004 & 2010 \\
\hline \multicolumn{4}{|c|}{ Ouvrages traduits en grec et en polonais } \\
\hline Paul Ricœur & Sur la traduction & 2004 & $\begin{array}{l}2008(\mathrm{gr}) \\
2010(\mathrm{pl})\end{array}$ \\
\hline \multicolumn{4}{|c|}{ III. OUVRAGES TRADUITS DE L'ALLEMAND } \\
\hline \multirow{2}{*}{ Auteur } & \multirow{2}{*}{ Titre(s) } & \multicolumn{2}{|c|}{ Année de la parution } \\
\hline & & original & traduction \\
\hline \multicolumn{4}{|c|}{ Ouvrages traduits en grec } \\
\hline Margret Ammann & $\begin{array}{l}\text { Grundlagen der modernen } \\
\text { Translationstheorie }\end{array}$ & 1990 & 2014 \\
\hline Walter Benjamin & Die Aufgabe des Übersetzers & 1923 & 2014 \\
\hline
\end{tabular}


Sur un tel fond se détachent les deux anthologies :

Tableau 6. Auteurs dont les textes se trouvent dans une anthologie

ANTHOLOGIES

(En gras, les noms des auteurs qui apparaissent dans les deux anthologies)

\begin{tabular}{|l|l|}
\hline \multicolumn{1}{|c|}{ Grèce } & \multicolumn{1}{c|}{ Pologne } \\
\hline & M. Heydel, P. Bukowski (dir.), Współczesne \\
D. Goutsos, O Logos tis Metafrasis (2005) & teorie przekładu (2009) \\
17 textes de : & 23 textes de : \\
Bankier, Chamberlain, Even-Zohar, & Berman, Chamberlain, Derrida, Even-Zohar, \\
Hermans, Heylen, Johnston, Keeley, Koller, & Gadamer, Hermans, Jakobson, Koller, \\
Lefevere, Nida, Robinson, Schmidt, Steiner & Lefevere, Levy, Neubert, Nida, Nord, Paepcke, \\
(3 textes), Venuti (2 textes) & Popovic, Ricœur, Riffaterre, Spivak, Steiner, \\
& Stolze, Toury, Tymoczko, Venuti \\
\hline
\end{tabular}

Ces recueils de textes - articles ou chapitres d'ouvrages d'auteurs reconnus - constituent une possibilité d'y accéder, et la seule en traduction. Leur importance indéniable dans le transfert des connaissances traductologiques est d'autant plus à souligner ${ }^{35}$.

On remarque qu'en Grèce les ouvrages traduits paraissent généralement chez des maisons d'édition généralistes, alors qu'en Pologne, il s'agit des presses universitaires ou spécialisées dans l'édition scientifique, à l'exception de l'anthologie polonaise qui a été publiée par un éditeur généraliste (la maison d'édition Znak de Cracovie). Une observation plus détaillée permet de constater, dans les deux pays, la dispersion des titres traduits entre divers éditeurs et dans des collections diverses (tableau 7). Aucun programme systématique et de longue haleine ne semble dicter les choix des éditeurs. En Grèce, la collection « Theoria ke praktiki tis metafrasis » ['Théorie et pratique de la traduction'] de la maison d'édition Metaichmio était prometteuse, mais elle s'est arrêtée en 2007 avec quatre titres ; l'éditeur Diavlos, qui à partir de 2007 a déjà publié quatorze titres en traductologie d'auteurs grecs et quatre traductions récentes des ouvrages de Michael Cronin, de Christiane Nord et de Margret Ammann semble ouvrir des perspectives intéressantes.

35 Ces anthologies mériteraient une étude à part ; au-delà de la question du statut même de l'anthologie (genre ou pratique discursive ?), il se poserait le problème de la représentation de la pensée traductive construite par les auteurs de ces «traductions groupées » (L. D'hulst, Essais d'histoire de la traduction, Classiques Garnier, Paris 2014, pp. 200-208). 
Tableau 7. Éditeurs des traductions

\begin{tabular}{|c|c|c|c|}
\hline \multicolumn{2}{|l|}{ Grèce } & \multicolumn{2}{|l|}{ Pologne } \\
\hline éditeur & $\begin{array}{l}\text { nombre } \\
\text { de titres } \\
\text { publiés }\end{array}$ & éditeur & $\begin{array}{l}\text { nombre } \\
\text { de titres } \\
\text { publiés }\end{array}$ \\
\hline Diavlos (à partir de 2007) & 4 & $\begin{array}{l}\text { Wydawnictwo Uniwersytetu } \\
\text { Gdańskiego, collection } \\
\text { «Europejscy klasycy sztuki } \\
\text { przekładu od XIV do XIX wieku » }\end{array}$ & 3 \\
\hline $\begin{array}{l}\text { Metaichmio (2002-2007), } \\
\text { collection « Theoria ke praktiki } \\
\text { tis metafrasis » }\end{array}$ & 4 & $\begin{array}{l}\text { Tertium, collection } \\
\text { « Język a komunikacja » }\end{array}$ & 4 \\
\hline Texto & 2 & $\begin{array}{l}\text { Universitas, collections : } \\
\text { « Horyzonty Nowoczesności », } \\
\text { « Językoznawstwo kognitywne », } \\
\text { "Międzykulturowe konteksty } \\
\text { kognitywizmu » }\end{array}$ & 3 \\
\hline Patakis & 2 & $\begin{array}{l}\text { Wydawnictwo Uniwersytetu } \\
\text { Jagiellońskiego, collection } \\
\text { «Translatio » }\end{array}$ & 2 \\
\hline Ellinika Grammata & 2 & Wydawnictwo Naukowe UAM & 1 \\
\hline divers éditeurs & $1 \times 10$ & $\begin{array}{l}\text { Wydawnictwa Naukowo-Tech- } \\
\text { niczne }\end{array}$ & 1 \\
\hline & & Wydawnictwo Literackie & 1 \\
\hline & & Wydawnictwo Znak & 1 \\
\hline
\end{tabular}

\subsection{BILAN}

La place de l'intraduction des ouvrages traductologiques est faible, voire très faible, dans les deux pays. On traduit à partir des langues considérées comme centrales, et notamment de l'anglais (et des auteurs anglo-saxons) - ce qui confirme la dominance de l'anglais comme langue des Translation Studies - et, dans un moindre degré, du français et de l'allemand. Le choix des titres semble très aléatoire et, aussi bien en Grèce qu'en Pologne, il serait impossible de parler d'un programme systématique et de longue haleine permettant l'intraduction des travaux majeurs de la traductologie. 


\section{USAGE DIDACTIQUE DES OUVRAGES TRADUCTOLOGIQUES TRADUITS EN GRÈCE ET EN POLOGNE}

\subsection{CONSTITUTION DES LISTES DE LECTURES CONSEILLÉES AUX ÉTUDIANTS}

Le but de notre travail étant de voir la part de la traduction dans la propagation des connaissances en traductologie, nous avons examiné les bibliographies qui accompagnent les programmes (syllabi) de divers cours de traduction/traductologie dispensés dans les universités grecques et polonaises.

Les différences de l'organisation des formations en traduction dans les deux pays ainsi que le statut des cours et matières qui les composent ont rendu difficile la création des listes des lectures conseillées aux étudiants qui présenteraient les mêmes caractéristiques. Il faut surtout souligner l'existence, en Grèce, d'un système universitaire de distribution gratuite des livres aux étudiants (Eudoxus) ${ }^{36}$ et d'une collection d'e-books académiques rédigés en langue grecque et disponibles en accès libre (Kallipos), qui peuvent être un canal important favorisant la transmission des savoirs. Le tableau 8 montre les sources utilisées, qui présentent des analogies qui nous semblent suffisantes pour mener des observations comparatives.

Pour dresser la liste des lectures obligatoires ou conseillées en Grèce, nous avons eu recours à deux sources : les sites web universitaires et la plateforme Eudoxus. À partir de la liste des départements de langues et littératures étrangères (français, anglais, allemand, italien, espagnol, russe, langues slaves, turc, langues balkaniques, études méditerranéennes) dans quatre universités différentes (NKUA, AUTH, Universités de Thrace et d'Égée), et du seul département spécialisé en traduction et interprétation (DFLTI), nous avons répertorié 21 cours obligatoires de $1^{\text {er }}$ cycle en traduction, appelés « introduction à la traduction », « théories de la traduction », « théorie et pratique de la traduction », etc.

Pour ces cours, nous avons recensé les ouvrages : (a) proposés dans les syllabi (disponibles dans les guides de l'étudiant de l'année académique 2018/2019 sur les sites web des départements) et/ou (b) distribués aux étudiants par le biais du système Eudoxus (dans les années académiques 2017/2018 et 2018/2019).

Quant aux cours de $2^{\mathrm{e}}$ cycle, pour lesquels Eudoxus n'est pas disponible, nos données sont très limitées, car nous n'avons trouvé que 3 syllabi, ceux des cours « principes de traductologie » (AUTH), « théorie et méthodologie de la traduction » (DFLTI), « théorie et pratique de la traduction » (NKUA) $)^{37}$.

${ }^{36}$ Un enseignant peut proposer pour son cours un à trois titres publiés par un éditeur grec et répertoriés sur la plateforme Eudoxus ; l'étudiant ne peut en choisir qu'un seul pour chaque cours. Tous les enseignants ne proposent pas d'ouvrage sur Eudoxus. La distribution aux étudiants est prise en charge par le Ministère de l'Éducation, ce qui constitue un motif pour faire publier des titres (originaux ou traduits) qui sont en relation avec les matières académiques enseignées.

37 Des séminaires de traduction sont également offerts dans le cadre des programmes autres que ceux de spécialisation en traduction ou en traductologie examinés ici ; notons, à titre indicatif, le séminaire de master « traduction théâtrale : théorie et pratique » du Département d'études théâtrales (NKUA). 
Pour dresser la liste des lectures obligatoires ou conseillées en Pologne, nous avons eu recours principalement au système USOS (Uniwersytecki System Obstugi Studiów - Système universitaire de la gestion des études), utilisé dans la plupart des universités polonaises, qui offre la possibilité d'accès à des syllabi de cours dispensés. Pour nos besoins, nous avons prélevé les bibliographies de 46 cours enseignés dans les années 2016/2017, 2017/2018, 2018/2019, dans les universités de Varsovie, Łódź, Wrocław, Jagellone (UJ), Śląski de Katowice, Marie Curie de Lublin, Adam Mickiewicz de Poznań (UAM), Mikołaj Kopernik de Toruń, Université Pédagogique de Cracovie, dans les cursus de deuxième cycle en anglais, français, allemand, espagnol, russe, et dans le cursus de deuxième cycle en polonais, spécialité traduction (UJ, UAM). Il s'agit de :

(1) Cours appelés « initiation à la traductologie», " éléments de traductologie» ou « théorie de la traduction »; ils sont proposés (a) dans le cadre d'un programme de formation à la traduction et/ou (b) dans un cursus « philologique » de premier (plus rare) ou de deuxième cycle en anglais, français, allemand, espagnol, russe, etc. Dans plusieurs cas, le même syllabus a été réutilisé, d'une année à l'autre ; pour les besoins de cette étude, il a été considéré une seule fois.

(2) Séminaires en traductologie, menant à la rédaction d'un mémoire consacré à la problématique de la traduction.

Tableau 8. Constitution des listes de lectures conseillées

\begin{tabular}{|c|c|c|}
\hline & Grèce & Pologne \\
\hline Années & $2017 / 2018,2018 / 2019$ & $2016 / 2017,2017 / 2018,2018 / 2019$ \\
\hline $\begin{array}{l}\text { Universités } \\
\text { et départe- } \\
\text { ments }\end{array}$ & $\begin{array}{l}13 \text { départements (de langues et litté- } \\
\text { ratures étrangères et de traduction/ } \\
\text { interprétation) }\end{array}$ & $\begin{array}{l}9 \text { universités (Varsovie, Jagellone, } \\
\text { Śląski, Wrocław, UMCS, UAM, UMK, } \\
\text { UPC, Łódź) }\end{array}$ \\
\hline \multirow[t]{2}{*}{ Sources } & $\begin{array}{l}\text { (1) sites web des départements } \\
\text { (2) Eudoxus }<\text { https://eudoxus.gr/> }\end{array}$ & USOS < https://www.usos.edu.pl/> \\
\hline & Grèce & Pologne \\
\hline Cursus & $\begin{array}{l}\text { (a) } 1^{\mathrm{er}} \text { cycle en anglais, français, } \\
\text { allemand, italien, espagnol, russe, turc, } \\
\text { langues balkaniques } \\
\text { (b) } 2^{\mathrm{e}} \text { cycle, spécialité traduction } \\
\text { (AUTH, NKUA, DLFTI) }\end{array}$ & $\begin{array}{l}1^{\mathrm{er}} \text { cycle (licencjat) en anglais et } \\
\text { français } \\
2^{\mathrm{e}} \text { cycle (magisterski) : } \\
\text { (a) en anglais, français, allemand, } \\
\text { espagnol, russe } \\
\text { (b) en polonais, spécialité traduction } \\
\text { (UJ, UAM) }\end{array}$ \\
\hline \multirow[t]{2}{*}{$\begin{array}{l}\text { Nombre de } \\
\text { cours }\end{array}$} & 24 syllabi & 46 syllabi \\
\hline & $\begin{array}{l}\text { liste grecque : } 108 \text { ouvrages } \\
\text { (dont l'anthologie) }\end{array}$ & $\begin{array}{l}\text { liste polonaise : } 243 \text { ouvrages } \\
\text { (dont l'anthologie) }\end{array}$ \\
\hline
\end{tabular}

Les syllabi contiennent aussi bien des livres que des articles, mais pour les besoins de ce travail seuls les travaux sous forme de livre ont été retenus. Par- 
mi ceux-ci apparaissent non seulement des ouvrages traductologiques, mais aussi ceux qui relèvent d'autres disciplines, par ex. histoire de la littérature, sémiotique, linguistique, sociologie. Leur élimination a permis d'obtenir des « listes brutes", qui sont une somme de toutes les entrées renvoyant à des ouvrages traductologiques dans les bibliographies réunies. Certains réapparaissent plusieurs fois, car ils figurent dans la plupart des syllabi examinés. Pour découvrir les ouvrages considérés par les enseignants grecs et polonais comme les plus significatifs pour la diffusion des connaissances en traductologie, le contenu des listes brutes a été ordonné selon la fréquence de chaque entrée ; ainsi, ont été obtenues les listes finales. Elles comptent : (a) les travaux des auteurs grecs/polonais publiés en grec/ polonais, (b) les travaux des auteurs grecs/polonais publiés en une langue étrangère, (c) les travaux des auteurs étrangers publiés en une langue étrangère et, enfin, (d) les travaux des auteurs étrangers traduits et publiés en grec/polonais.

\subsection{RÉSULTATS DE L'EXAMEN DES LECTURES CONSEILLÉES}

Les données réunies ainsi peuvent être considérées comme une indication des tendances qui existent dans les deux pays quant à la part de l'intraduction dans la transmission didactique des connaissances en traductologie.

Ainsi, les graphiques $3 \mathrm{a}, 3 \mathrm{~b}$ et $3 \mathrm{c}$ montrent la présence plus forte des ouvrages traduits sur la liste grecque (ceci n'étonne pas si l'on rappelle que le nombre de traductions en grec est légèrement plus important et que grâce au système Eudoxus sont proposés aux étudiants les livres publiés chez des éditeurs grecs, dont ceux qui sont traduits en grec) et le nombre plus important d'ouvrages originaux en langue étrangère sur la liste polonaise.

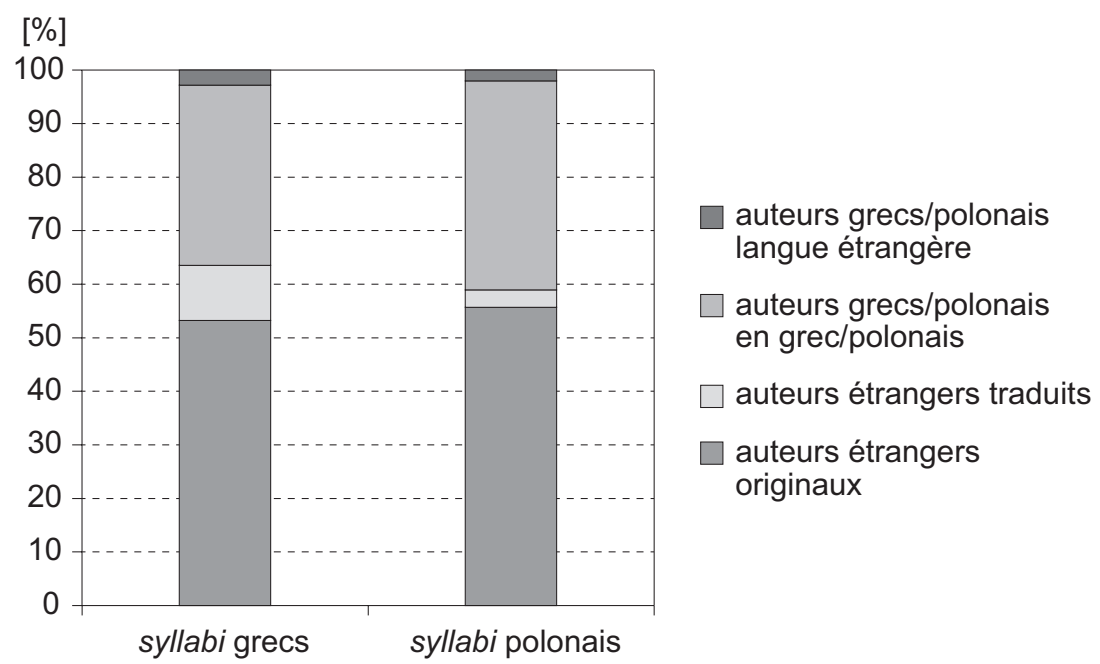

Graphique 3a. Nombre d'ouvrages originaux et en traduction dans les syllabi grecs et polonais 


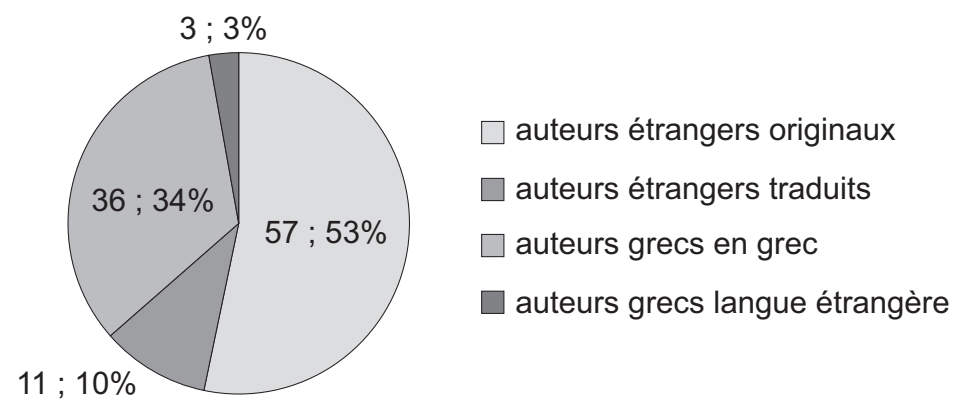

Graphique 3b. Nombre d'ouvrages originaux et en traduction dans les syllabi grecs

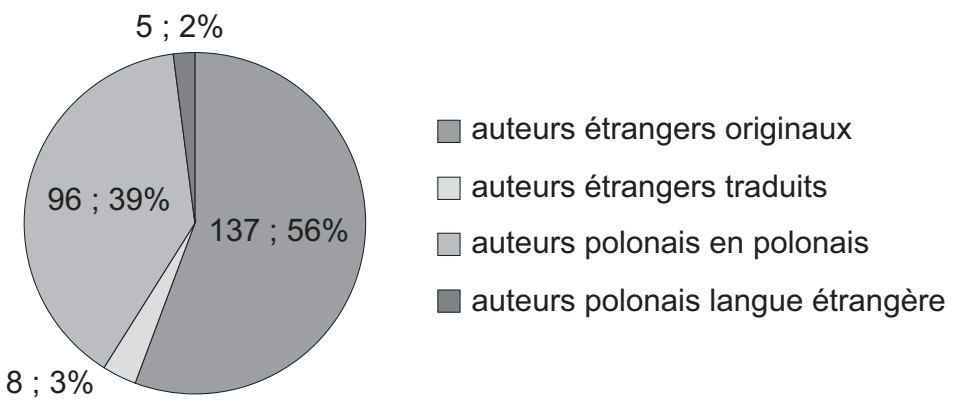

Graphique 3c. Nombre d'ouvrages originaux et en traduction dans les syllabi polonais

L'approche plus détaillée, concernant la langue originale de l'ouvrage (graphiques $4 \mathrm{a}$ et $4 \mathrm{~b}$ ), montre la dominance de l'anglais dans les deux listes (43 originaux et 4 traductions en grec ; 84 originaux et 5 traductions en polonais) et une présence plus forte du français dans la catégorie « ouvrages traduits » dans la liste grecque (5 en grec contre 1 en polonais), ce qui correspond aux nombre plus important de traductions, que nous avons vu auparavant.

Si la position de l'anglais n'étonne pas, on peut être frappé par le nombre bas des lectures en allemand ; dans leur article de 2005, Balacescu et Stefanink déplorent la méconnaissance des apports théoriques de la traductologie allemande ${ }^{38}$ : nos données semblent le confirmer, surtout pour la Pologne. Et comme les travaux allemands ne sont pas traduits — nous l'avons vu plus haut — cette situation a peu de chances de changer.

${ }^{38}$ I. Balacescu, B. Stefanink, op. cit., p. 278. 


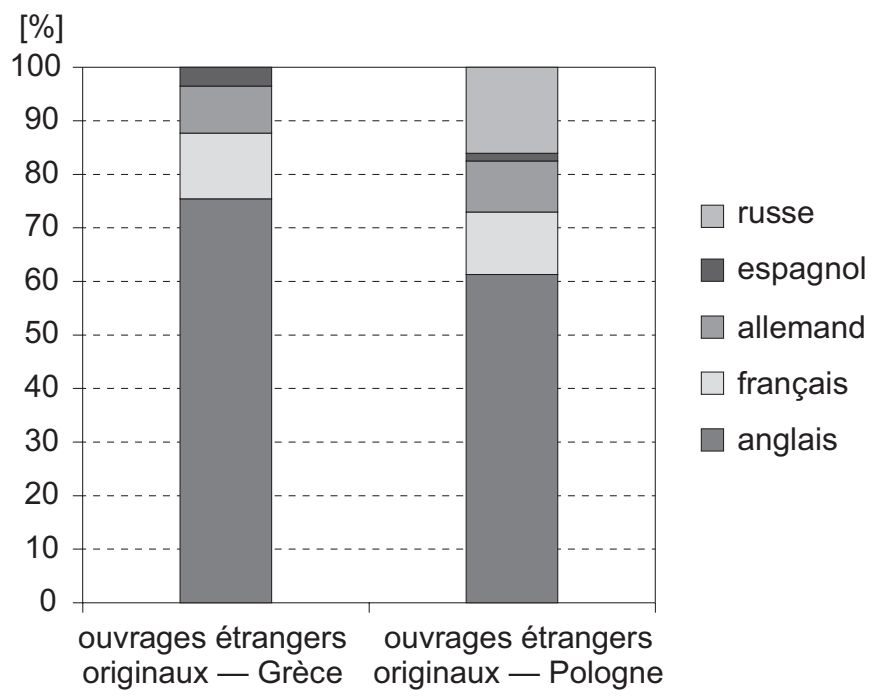

Graphique 4a. Nombre d'ouvrages étrangers originaux selon la langue, dans les syllabi grecs et polonais

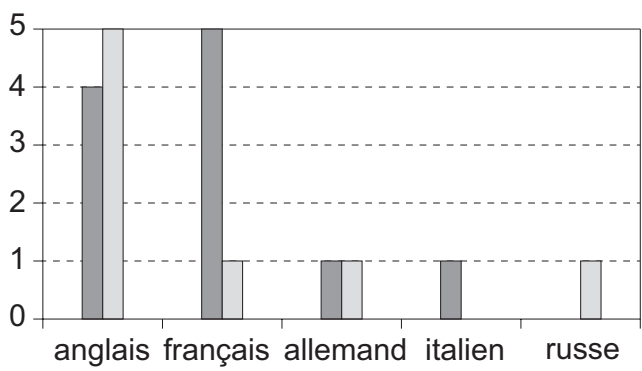

ouvrages étrangers traduits - Grèce

$\square$ ouvrages étrangers traduits - Pologne

Graphique 4b. Nombre d'ouvrages étrangers traduits selon la langue, dans les syllabi grecs et polonais

Le tableau 9 permet de faire des comparaisons concernant la place des ouvrages traduits dans les syllabi. Ainsi, si en Grèce seuls sept titres traduits y apparaissent (sur 24), neuf (sur 18) sont cités dans la liste polonaise. Mais c'est surtout leur fréquence qui attire l'attention. Dans la liste grecque, c'est le manuel de Jeremy Munday qui est le plus cité, avec onze mentions dans les syllabi (et quinze dans les listes destinées à la distribution via Eudoxus) ; ensuite vient le fonctionnalisme allemand avec le manuel de Nord ; avec trois mentions apparaissent des ouvrages à caractère plus théorique et qui peuvent être traités de "classiques ", si l'on prend en considération l'année de la publication de l'original : Belles infidèles de Mounin (1955) et Théorèmes pour la traduction de Ladmiral (1979). 
On y voit aussi Terminologie de la traduction (deux mentions), le seul ouvrage figurant dans les deux listes et qui, avec quatorze mentions, apparaît en tête de la liste polonaise. Sur celle-ci, le deuxième ouvrage le plus souvent cité est l'anthologie, mentionnée onze fois - alors que l'anthologie grecque n'apparaît qu'une seule fois. Quant aux autres titres traduits, ils sont mentionnés une à deux fois. Ceci semble indiquer plutôt les prédilections des enseignants que la valeur ou la place des ouvrages dans la pensée traductologique ${ }^{39}$.

Tableau 9. Usage didactique des ouvrages traduits

\begin{tabular}{|c|c|c|c|}
\hline \multirow[t]{3}{*}{ Ouvrages traduits } & \multicolumn{3}{|c|}{ Nombre d'apparitions sur les listes } \\
\hline & \multicolumn{2}{|c|}{ grecque } & \multirow{2}{*}{$\begin{array}{c}\text { polonaise } \\
\text { syllabi }\end{array}$} \\
\hline & syllabi & Eudoxus & \\
\hline Delisle et al. (Terminologie de la traduction) & 2 & 0 & 14 \\
\hline Cronin (Translation in the Digital Age) ${ }^{40}$ & 0 & 0 & 2 \\
\hline Steiner (After Babel) & 0 & 0 & 2 \\
\hline Ricœur (Sur la traduction) & 0 & 0 & 1 \\
\hline Anthologie (Współczesne teorie przekładu) & & & 11 \\
\hline $\begin{array}{l}\text { Tabakowska (Cognitive Linguistics and Poetics of Trans- } \\
\text { lation) }\end{array}$ & & & 2 \\
\hline Dedecius (Notatnik tlumacza) & & & 2 \\
\hline Torop (Total'nyj perevod) & & & 1 \\
\hline Hermans (The Conference of the Tongues) & & & 1 \\
\hline Munday (Translation Studies) & 11 & 15 & \\
\hline Nord (Translating as a Purposeful Activity) & 2 & 8 & \\
\hline Ladmiral (Traduire : théorèmes pour la traduction) & 3 & 0 & \\
\hline Mounin (Les problèmes théoriques de la traduction) & 0 & 4 & \\
\hline Mounin (Les belles infidèles) & 3 & 0 & \\
\hline Cronin (Translation and Globalization) & 0 & 2 & \\
\hline Gouadec (Profession : Traducteur) ${ }^{41}$ & $1(1)$ & 0 & \\
\hline Anthologie (O Logos tis Metafrasis) & 1 & 0 & \\
\hline
\end{tabular}

${ }^{39}$ Certains écarts entre les deux listes peuvent peut-être s'expliquer par le fait que le système grec est directement lié aux maisons d'éditions et au stock disponible (par ex. l'éditeur de l'anthologie a fermé en 2010 et la Terminologie est épuisée chez l'éditeur). Cette conjecture nécessiterait cependant une recherche plus détaillée sur le marché éditorial de chaque pays.

40 La traduction grecque, publiée en novembre 2019, n'entre pas dans la période examinée ici.

${ }^{41}$ Ce titre apparaît une fois en traduction grecque et une fois en traduction anglaise. 


\subsection{BILAN}

L'examen des listes des lectures dévoile un intérêt très faible que portent les enseignants à des ouvrages traduits. Leur place dans les syllabi - que l'on peut considérer comme une sorte de canon de base - est infime, en comparaison à celle des ouvrages étrangers originaux : plus de 50\% dans les deux pays, nombre supérieur à celui des ouvrages écrits en langue maternelle (30\% environ). Un seul titre apparaît plus de dix fois sur la liste grecque, deux dans les syllabi polonais considérés comme un tout. Dans tous les cas, il s'agit d'ouvrages à caractère didactique (manuel, dictionnaire, anthologie) ; ceci n'étonne pas, la majorité des cours étant ceux d'initiation à la traductologie. La très faible présence ou l'absence des ouvrages de caractère plus théorique (Steiner, Ricœur, Mounin) peut s'expliquer par le fait qu'ils peuvent être utilisés surtout en séminaires qui préparent à la recherche ; or ceux-ci ne constituent qu'une petite partie des cours dont les syllabi ont été utilisés.

\section{CONCLUSION}

Nos observations apportent une réponse négative à la question liminaire : la traduction ne sert que dans une très faible mesure à propager les connaissances en traductologie, aussi bien en Grèce qu'en Pologne. Pour ce qui des ouvrages intraduits, non seulement leur nombre est infime, mais de plus le choix des titres ne semble pas être régi par un principe cohérent. Quant à leur usage didactique, il apparait être minime dans les cours universitaires. Dans les deux pays, la présence très forte d'ouvrages en langues étrangères dans les syllabi — qui reflète, pourrait-on croire, les « préférences » des enseignants-chercheurs — contribue à la pérennisation du phénomène du « séparatisme linguistique » et de la domination de la pensée traductologique anglosaxonne.

La suprématie de la traductologie anglosaxonne se manifeste également par l'absence de contact avec les " périphéries ": à part Ljudskanov en Pologne (1973), aucun ouvrage traductologique n'a été intraduit d'une langue périphérique, ni en grec, ni en polonais ; et il en va de même pour l'extraduction des ouvrages grecs et polonais vers d'autres langues ${ }^{42}$. De plus, les travaux de chercheurs de pays et langues « périphériques », même ceux publiés en anglais $(\mathrm{Zu}-$ zana Jettmarova, Kaisa Koskinen, Niki Pokorn — pour ne citer que quelques

${ }^{42}$ La recherche sur les flux de traductions montre l'absence totale de l'extraduction des ouvrages traductologiques du grec et quelques exceptions seulement pour le polonais : une traduction en italien (W. Soliński, Traduzione artistica e cultura letteraria: comunicazione e metacomunicazione letteraria, trad. F. Tucci, Schena Editore, Fasano 1992), deux autres en préparation grâce à la subvention de l'EST $(<$ https://est-translationstudies.org/committees/translation-committee/translation-prize/ $>$ ) et l'anthologie de Bończa Bukowski et Heydel Polish Translation Studies in Action ..., sortie en 2019. 
noms), sont, eux aussi, absents des listes recensées. La phrase de Pierre Bourdieu, citée en exergue, semble donc trouver une confirmation de plus dans les cas grec et polonais.

Nos constatations invitent à de nouveaux questionnements. Le premier porte sur les raisons d'un tel état de choses dans les deux pays : pourquoi la plupart des ouvrages sont-ils traduits de l'anglais - langue que tous les chercheurs peuvent lire ? Quel est la part d'influence de l'université et des chercheurs ${ }^{43}$ auprès des différents acteurs qui participent à la dissémination des connaissances à travers la traduction : auteurs, éditeurs, traducteurs ? Cette situation présente-t-elle des analogies avec d'autres pays? Quel peut être en définitive le rôle du «multilinguisme passif » dans la propagation équitable des savoirs ?

Si ces questionnements ne se limitent pas au champ de la traductologie, c'est pourtant son rôle, comme le souligne Rafael Schögler, de montrer que la traduction « forme et transforme les champs académiques, culturels et intellectuels autant que leurs acteurs et les interprétations des idées $»^{44}$.

Et, ajoutons, de le faire par son propre exemple. En effet, il faut tenir compte d'un facteur de poids : si la reconnaissance institutionnelle de la traductologie comme discipline universitaire et domaine de connaissance est récente ${ }^{45}$, son développement a un caractère " transnational » : non seulement son objet, la traduction, incite à dépasser les frontières linguistiques et géographiques, mais aussi la recherche (et la publication des résultats) se fait souvent au-delà de ces frontières ${ }^{46}$.

Dans ce sens, il serait indispensable de reconsidérer comment se dessinent les centres et les périphéries, mais aussi quelles sont les véritables barrières à franchir (culturelles ? idéologiques? économiques ? politiques ?) pour ouvrir ou faciliter l'accès aux apports significatifs en traductologie.

${ }^{43}$ Qui se situent toujours dans un contexte sociopolitique national et international — facteur à ne pas oublier.

44 R. Schögler, « Les fonctions de la traduction en sciences humaines et sociales », Parallèles 29(2), octobre 2017, p. 37.

$45 C f$. Y. Gambier, «Institutionalization of translation studies », [dans :] L. D’hulst, Y. Gambier (dir.), A History of Modern Translation Knowledge..., pp. 179-194.

46 En 2009, Gideon Toury a observé un phénomène de migration académique, en examinant les affiliations des auteurs publiant dans Target - International Journal of Translation Studies (c'est nous qui soulignons), dont un nombre important travaillent dans un pays autre que celui de leur nationalité (G. Toury, "Incubation, birth and growth. Observations on the first 20 years of Target », Target 21(2), 2009, pp. 194-195). Aujourd'hui, à cette migration s'ajoutent les politiques de recherche et la coopération internationale qui encouragent la mobilité des chercheurs et la publication de leurs travaux en langues autres que leur langue maternelle ; $c f$. aussi l'article de Kristiina Taivalkoski-Shilov dans ce volume. 


\section{ANNEXES}

\section{CORPUS D'ANALYSE GREC}

Ammann M., Vasikes arches tis metafraseologias [Grundlagen der modernen Translationstheorie], trad. A. Wiedenmayer, D. Lamprou, Diavlos, Athènes 2014.

Benjamin W., I apostoli tou metafrasti [Die Aufgabe des Übersetzers], trad. G. Sagkriotis, Patakis, Athènes 2014.

Berman A., I metafrasi ke to gramma i To pandochio tou apomakrou [La traduction et la lettre ou L'auberge du lointain], trad. C. Inglessis Margellos, Metaichmio, coll. «Theoria ke praktiki tis metafrasis ", Athènes 2002.

Christensen B., Liedtke K.-J., Sta kimata trion thalasson: mia synchroni Odysseia [Waves of Three Seas: A modern Odyssey], trad. Th. Liveriadis, International Writers and Translators' Center of Rhodes, Rhodes 1996.

Cronin M., Metafrasi ke pagkosmiopiisi [Translation and Globalization], trad. P. Kelandrias, Diavlos, Athènes 2007.

Cronin M., I metafrasi stin psifiaki epochi [Translation in the Digital Age], traduit par les étudiants en Master «Traduction-Traductologie » NKUA, M. Pantazara (dir.), Diavlos, Athènes 2019.

Delisle J., Lee-Jahnke H., Cormier M.C., Orologia tis metafrasis [Terminologie de la traduction], trad. G. Floros, Mesogios, Athènes 1999.

Derrida J., Martyria ke metafrasi: epivionontas piitika. Ke tesseris anagnosis, trad. V. Bitsoris, Institut français d'Athènes, Athènes 1996.

Eco U., Empiries metafrasis [Experiences in Translation], trad. E. Kallifatidi, Ellinika Grammata, Athènes 2003.

Gentzler E., Sinchrones theories metafrasis [Contemporary Translation Theories], trad. Ch. Bakoula, Ion, Athènes 2012.

Gouadec D., Thelo mia metafrasi [Faire traduire], trad. R. Margaritari, Texto, Athènes 2010.

Gouadec D., Epaggelma metafrastis [Profession: Traducteur], trad. E. Kalogianni, Texto, Athènes 2008.

Goutsos D. (dir.), O Logos tis Metafrasis - Anthologio synchronon metafrastikon theorion, Ellinika Grammata, Athènes 2005.

Jakobson R., Dokimia gia ti glossa tis logotechnias [Language in Literature], trad. A. Berlis, Hestia, Athènes 1998.

Kelly N., Zetzsche J., Gia na min imaste chameni sti metafrasi [Found in Translation], trad. E. Tziafa, Pedio, Athènes 2017.

Ladmiral J.-R., Theorimata gia ti metafrasi [Traduire : théorèmes pour la traduction], trad. C. Collette, M.-C. Anastassiadi, Metaichmio, coll. « Theoria ke praktiki tis metafrasis », Athènes 2007.

Mounin G., I orees apistes [Les belles infidèles], traduit par les étudiants en Master « TraductionTraductologie » NKUA, A. Filippatos (dir.), Metaichmio, coll. « Theoria ke praktiki tis metafrasis », Athènes 2002.

Mounin G., Ta theoritika provlimata tis metafrasis [Les problèmes théoriques de la traduction], trad. I. Papaspyridou, Travlos, Athènes 2002.

Munday J., Metafrastikes spoudes — Theories ke efarmoges [Introducing Translation Studies], trad. A. Filippatos, Metaichmio, coll. « Theoria ke praktiki tis metafrasis », Athènes 2001.

Nord Ch., I metafrasi os stochevmeni drastiriotita [Translating as a Purposeful Activity], trad. S. Grammenidis, D. Lamprou, Diavlos, Athènes 2014.

Ricœur P., Gia ti metafrasi [Sur la traduction], trad. G. Avgoustis, Patakis, Athènes 2010.

Schleiermacher F., Peri ton diaforon methodon tou metafrazein [Über die verschiedenen Methoden des Übersetzens], trad. K. Kotsiaros (édition bilingue), Gutenberg, Athènes 2014. 
Steiner G., Meta ti Vavel [After Babel], trad. G. Kondylis, Scripta, Athènes 2004.

Williams J., Chesterman A., O chartis - I erevna stis metafrastikes spoudes [The Map-A. Beginner's Guide to Doing Research in Translation Studies], traduit par les étudiants en Master « TraductionTraductologie » NKUA, M. Sidiropoulou (dir.), Ypsilon, Athènes 2010.

\section{CORPUS D’ANALYSE POLONAIS}

Arnold M., O przektadaniu Homera [On Translating Homer], trad. K.F. Rudolf, Wydawnictwo Uniwersytetu Gdańskiego, coll. «Europejscy klasycy sztuki przekładu od XIV do XIX wieku », Gdańsk 2018.

Bukowski P., Heydel M. (dir.), Wspótczesne teorie przekładu: antologia, Wydawnictwo Znak, Kraków 2009.

Cronin M., Przeklad w epoce cyfrowej [Translation in the Digital Age], trad. M. Błaszkowska, M. Heydel, E. Koziołkiewicz, M. Nawrocki, A. Pikul, Z. Ziemann; sous la direction de M. Heydel et Z. Ziemann, Wydawnictwo Uniwersytetu Jagiellońskiego, Kraków 2016.

Dedecius K., Notatnik thumacza, trad. J. Prokop, I. et E. Naganowscy, Wydawnictwo Literackie, Kraków 1974.

Delisle J., Lee-Jahnke H., Cormier M.C. (dir.), Terminologia tłumaczenia [Terminologie de la traduction], traduction et adaptation T. Tomaszkiewicz, Wydawnictwo Naukowe UAM, Poznań 2004.

Fraser Tytler A. (lord Woodhouselee), Esej o zasadach sztuki przekładowczej [Essay on the Principles of Translation], trad. K.F. Rudolf, W. Waśniewska, Wydawnictwo Uniwersytetu Gdańskiego, coll. « Europejscy klasycy sztuki przekładu od XIV do XIX wieku », Gdańsk 2014.

Gillies A., Ttumaczenie ustne: poradnik dla studentów [Conference Interpreting: A New Students' Companion], B. Waliczek (dir.), traduction de la partie polonaise A. Jagoda et M. Piechaczek, Tertium, Kraków 2001.

Gillies A., Sztuka notowania: poradnik dla thumaczy konferencyjnych, trad. A. Wyrwa, Tertium, Kraków 2007.

Gutt E.-A., Dystans kulturowy a przekład [Modes of Translation and Cultural Distance], trad. A. Pokojska, Universitas, coll. « Międzykulturowe konteksty kognitywizmu », Kraków 2004.

Hermans T., Narada języków [The Conference of the Tongues], trad. A. Dauksza, M. Heydel, K. Michalik, M. Pitek, K. Rozwadowska, K. Szymańska, K. Wasilewska, K. Wojda; sous la direction de M. Heydel et K. Szymańska, Wydawnictwo Uniwersytetu Jagiellońskiego, Kraków 2015.

Ludskanow A., Ttumaczy człowiek i maszyna cyfrowa [Preveždat čovek"t i mašinata], trad. K. Leski, A. Naumow, Wydawnictwa Naukowo-Techniczne, Warszawa 1973.

Newman F., Teoria i praktyka przekladu Homera [Homeric Translation in Theory and Practice: a Reply to Matthew Arnold], trad. K.F. Rudolf, Wydawnictwo Uniwersytetu Gdańskiego, coll. « Europejscy klasycy sztuki przekładu od XIV do XIX wieku», Gdańsk 2018.

Ricœur P., Torop P., O tlumaczeniu / Przeklad całkowity [Sur la traduction / Total'nyj perevod], trad. T. Swoboda, S. Ulaszek, Wydawnictwo Uniwersytetu Gdańskiego, Gdańsk 2008.

Rozan J.-F., Notatki w tlumaczeniu konsekutywnym [Prise de notes en interprétation consécutive], trad. U. Hrehorowicz, Tertium, coll. « Język a komunikacja », Kraków 2002.

Steiner G., Po wieży Babel: problemy języka i przekładu [After Babel], trad. O. et W. Kubińscy, Universitas, coll. «Horyzonty Nowoczesności », Kraków 2000.

Tabakowska E., Językoznawstwo kognitywne a poetyka przekladu [Cognitive Linguistics and Poetics of Translation], trad. A. Pokojska, Universitas, coll. « Językoznawstwo kognitywne », Kraków 2001. 


\title{
CAN TRANSLATION BE A MEANS OF KNOWLEDGE DISSEMINATION IN TRANSLATION STUDIES? GREECE AND POLAND AS CASE STUDIES
}

\begin{abstract}
The aim of this paper is to examine the factors that may boost or hinder the effectiveness of translation as a vehicle of knowledge dissemination. The analysis was based on bibliographical data concerning the translations of books of all kinds (monographs, handbooks, textbooks, anthologies, etc.) in the field of translation studies, which have been published in Greece and Poland. Both countries can be considered as being on the periphery of Europe on the basis of geographical, political, economic, linguistic, and cultural criteria. In both countries, the number of these translations is actually very small (about 20 in each of them), and the number of their source languages is extremely limited (mostly English, rarely French or German). However, the different organization of their universities and academic research shapes not only how the individual translation training and scientific work on translation are developed, but also how these translations are embedded in university syllabi. The analysis of the data leads to confirming the dominance of English as the translation studies language and suggests a necessity to reconsider the notions of centres and peripheries in this particular field of research and the role of translation in their relationship.
\end{abstract}

Key words: translation studies, translation, knowledge dissemination, Greece, Poland. 